\title{
TEM and SAXS Investigation of Self-Assembled 2D Structures of PbS Quantum- Dots Deposited onto Different Underlying Substrates
}

\author{
Said A. Mansour ${ }^{1}$, Dhanasekaran Thirunvukka ${ }^{1}$ and Brahim Aïssa ${ }^{1}$ \\ 1. Qatar Environment and Energy Research Institute, Hamad Ben Khalifa University, Qatar Foundation, \\ Doha, Qatar.
}

Colloidal semiconductor nanocrystals (NCs) are widely explored as functional materials for photonics and energy applications including solar cells, light emitting diodes, photodetectors, field-effect transistors, memory elements and thermoelectric elements. Key properties of NCs revolve around synthesis methods, solution processability, high-photoluminescence quantum yield, good photo-stability, and the possibility to control their electronic structure by engineering their composition, size, and shape. Among various kinds of NCs, inorganic lead sulfide quantum dots (QD) have tunable band gap and high absorption coefficients suitable for harvesting a large fraction of solar spectrum photons. Moreover, $\mathrm{Pb}$ and $\mathrm{S}$ are major industrial elements with suitable natural abundance for large-scale production. Our interest in $\mathrm{PbS}$ is to enhance the solar energy conversion process as it is desirable to extend the light absorption edge of the photo-electrodes.

In this work, we present a systematic analysis of the structural and morphological properties of selfassembled lead sulfide $(\mathrm{PbS})$ quantum-dots (PbS-QDs) in super lattice like structure in the 2D arrangement. High resolution transmission electron microscopy (HRTEM) using FEI TALOS X, and small-angle X-ray scattering (SAXS) using Rigaku S- Max 3000 were used to investigate the assembly trends of PbS-QDs attempted on different substrates. PbS-QDs of $1200 \mathrm{~nm}$ PL emission peak encapsulated in Oleic Acid (OA) and suspended in toluene were used. Samples for TEM imaging were prepared by depositing a small drop of diluted nanoparticles solution onto Lacy/ Carbon thin film on copper grid and allowed to evaporate. Samples for SAXS analysis were prepared by depositing a drop of nanoparticles solution onto Kapton tape and allowed to evaporate (Kapton tape is highly transparent to X-rays in the SAXS region).

Figure 1 (a) shows representative obtained TEM micrographs of the PbS-QDs exhibiting different orientation self-assembled super lattice (SL) domains. Processed fast Fourier transform (FFT) insets of neighboring domains suggested dominant 011 Face-Centered Cubic (FCC) SL structure with fewer domains exhibiting 001 FCC orientation. Close examination and spread of different FFT diffraction spots suggested imperfect super lattice exhibiting $\sim 10 \%$ distortions from parameters of perfect FCC assembly. Simple calculation of QD diameter corresponding to $6.20 \mathrm{~nm}$ distance between (001) SL planes led to $\mathrm{PbS}$ QDs diameter of $\sim 4.38 \mathrm{~nm}$.

High angle annular dark field (HAADF) image in Figure 1 (b) and corresponding diffraction pattern (DP) displayed in the inset show both high $2 \theta$ values as well as the low $2 \theta$ values of QDs super lattice. High angle diffraction exhibited (111) and (200) reflections, suggesting (011) dominant PbS crystalline orientation with preferential orientation. Super lattice suggested 001 orientation which is heavily dependent on orientation and ordering within assemblies of individual monodispersed faceted nanoparticles within the super lattice [1]. The FCC SL orientation and QDs sizes were further confirmed by SAXS analysis of deposited QDs on Kapton tape as shown in Fig. 2. The FCC lattice is assigned to low angle peaks was based on the $200 / 111$ peak intensity ratio giving a value of $\sim 0.866(\sqrt{ } 3 / 2)$ which is characteristic for the FCC peak positions. Average QDs diameter was calculated to be $4.33 \mathrm{~nm}$ in agreement with TEM calculated values. 
PbS-QDs of $1400 \mathrm{~nm}$ PL emission peak were also studied and will be presented. The effect of PbS QDs sizes, orientation and faceting on SL ordering, orientation and preferential bonding between adjacent NCs were studied and will also be discussed.

\section{References:}

[1] P Simon et al., Advanced Materials (2014), p. 1 http://sci-hub.tw/10.1002/adma.201305667
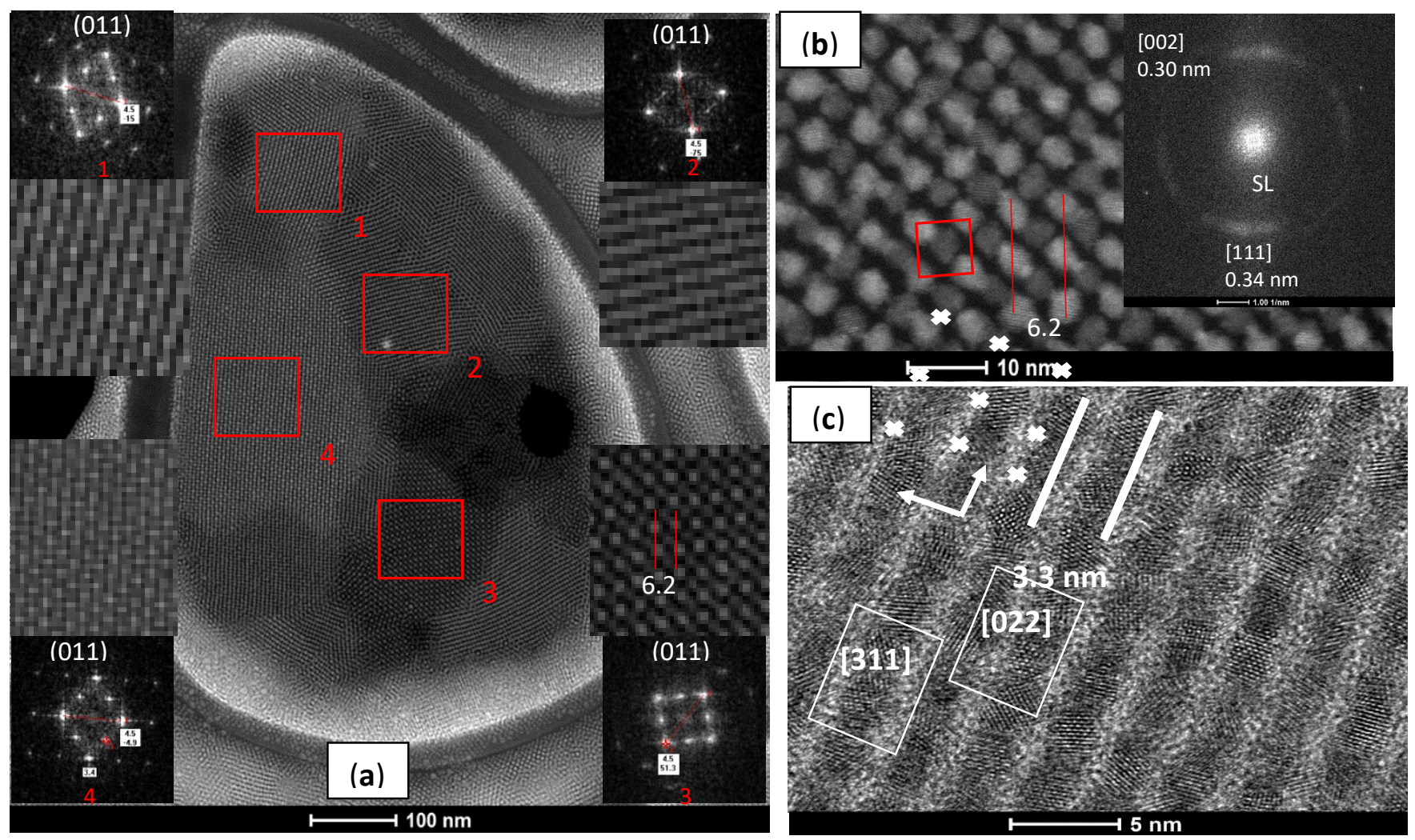

Figure 1: TEM, HAADF, HRTEM and processed FFT images showing (a) different PbS QD ordering domains within the self-assembled PbS-oleic acid monolayer, (b) Showing HAADF image of 001 SL while SAD shows high and low $2 \theta$ angle reflections representing PbS QD crystal lattice and SL plane, respectively, (c) HRTEM showing $\mathrm{SL}$ and random $\mathrm{PbS}$ QDs orientations in (011) SL plane
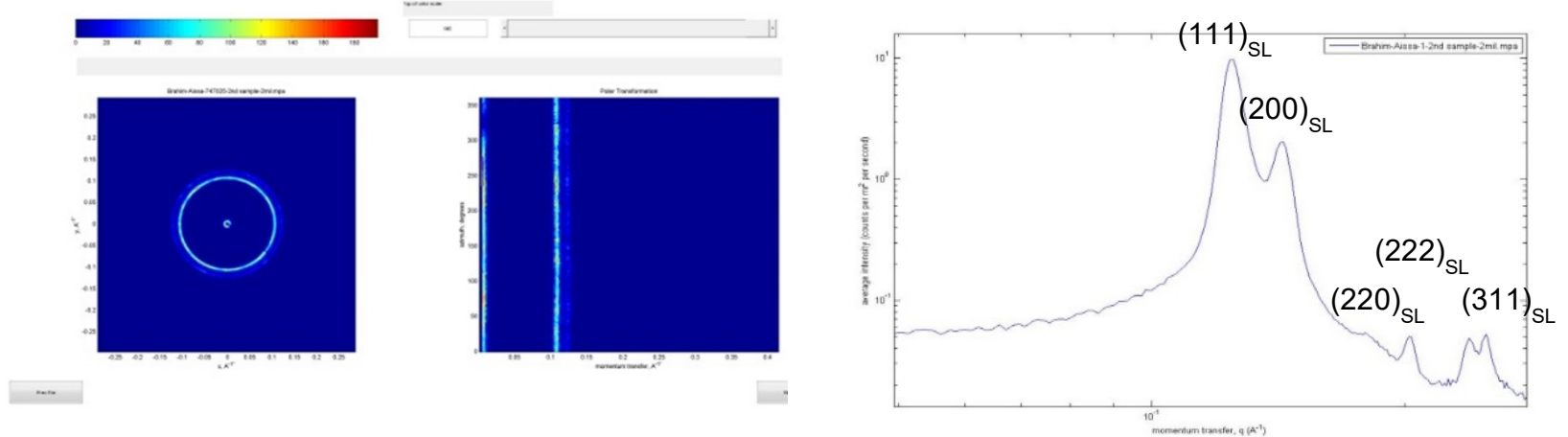

Figure 2: Angular and linear representation of PbS QD SAXS diffraction patterns on Kapton tape indicating random $\mathrm{PbS}$ QD distribution with strong 111 and 200 reflections. The FCC lattice was assigned based on ratio of 200/111 peak intensities. 